\title{
Graph-Theoretic Analysis of Epileptic Seizures on Scalp EEG Recordings
}

\author{
Nimit Dhulekar * \\ Basak Oztan, PhD. ** \\ Bülent Yener, PhD. \\ Department of Computer \\ Science \\ Rensselaer Polytechnic \\ Institute \\ Troy, NY, USA \\ dhulena@cs.rpi.edu \\ oztanb@cs.rpi.edu \\ yener@cs.rpi.edu
}

\author{
Haluk O. Bingol, PhD. \\ Dept. of Computer Eng. \\ Bogazici University \\ Istanbul, Turkey \\ bingol@boun.edu.tr
}

\author{
Gulcin Irim, \\ Berrin Aktekin, MD. \\ Canan Aykut-Bingöl, MD. \\ Dept. of Neurology \\ Yeditepe University \\ Istanbul, Turkey \\ girim@yeditepe.edu.tr \\ berrin.aktekin@yeditepe.edu.tr \\ caykutbingol@yeditepe.edu.tr
}

\begin{abstract}
This work presents a novel modeling of neuronal activity of the brain by capturing the synchronization of EEG signals along the scalp. The pair-wise correspondence between electrodes recording EEG signals are used to establish edges between these electrodes which then become the nodes of a synchronization graph. As EEG signals are recorded over time, we discretize the time axis into overlapping epochs, and build a series of time-evolving synchronization graphs for each epoch and for each traditional frequency band.

We show that graph theory provides a rich set of graph features that can be used for mining and learning from the EEG signals to determine temporal and spatial localization of epileptic seizures. We present several techniques to capture the pair-wise synchronization and apply unsupervised learning algorithms, such as $k$-means clustering and multiway modeling of third-order tensors, to analyze the labeled clinical data in the feature domain to detect the onset and origin location of the seizure. We use $k$-means clustering on two-way feature matrices for detection of seizures, and Tucker3 tensor decomposition for localization of seizures. We conduct an extensive parametric search to determine the best configuration of the model parameters including epoch length, synchronization metrics, and frequency bands, to achieve the highest accuracy.

Our results are promising: we are able to detect the onset of seizure with an accuracy of $88.24 \%$, and localize the onset of the seizure with an accuracy of $76.47 \%$.
\end{abstract}

\footnotetext{
${ }^{*}$ These authors contributed equally to this work.

* Dr. Oztan is now with American Science and Engineering, Billerica, MA 01821.
}

Permission to make digital or hard copies of part or all of this work for personal or classroom use is granted without fee provided that copies are not made or distributed for profit or commercial advantage and that copies bear this notice and the full citation on the first page. Copyrights for third-party components of this work must be honored. For all other uses, contact the Owner/Author.

BCB'14, September 20-23, 2014, Newport Beach, CA, USA.

Copyright is held by the owners/author(s)

ACM 978-1-4503-2894-4/14/09

http://dx.doi.org/10.1145/2649387.2649423.

\section{Categories and Subject Descriptors}

G.2 [Discrete Mathematics]: Graph Theory

\section{General Terms}

Algorithms, Measurement

\section{Keywords}

Epileptic seizures, EEG recordings, seizure detection, seizure localization, synchronization graphs, feature extraction, unsupervised learning, tensor decomposition

\section{INTRODUCTION}

Epilepsy is a common chronic neurological disorder that affects the nervous system and is typically characterized by the recurrent seizures caused by sudden bursts of electrical energy in the brain. For a successful treatment and understanding of the seizures, it is critical to characterize the physiological changes caused by the seizures.

Clinical evaluation, identification of epileptic seizures, and localization of epileptic seizures significantly rely on monitoring and analysis of long-term electroencephalographic (EEG) signals and benefit from intensive video-EEG monitoring. Large numbers of multi-channel EEG signals are visually analyzed by neurologists with a goal of understanding when and where the seizures start and how they propagate within the brain. However, there are two main disadvantages of visual analysis of EEG signals: it is time-consuming and prone to subjectivity. Therefore, automation of the detection of the underlying brain dynamics in EEG signals is significant in order to obtain fast and objective EEG analysis.

\subsection{Related Work}

A common approach in seizure recognition/detection and also in prediction is to extract information, e.g., features that can characterize seizure morphologies, from EEG recordings $[27,30,32,35,42]$. The procedure for feature extraction from multi-channel EEG data is often as follows: First, an EEG signal from a channel is divided into $I$ time epochs (overlapping or non-overlapping) and then $J$ features are 
extracted from each epoch. Consequently, a signal from a single channel can be represented as a matrix of size $I \times J$. A great deal of effort from different disciplines has been invested in exploring the features in order to capture characteristics of an epileptic seizure. These features include statistical complexity measures (e.g., fractal dimension, approximate entropy, Lyapunov exponents, etc.) as well as other features from time (e.g., higher-order statistics of the signal in time domain, Hjorth parameters, etc.) and frequency domains (e.g., spectral skewness, spectral entropy, etc.). A list of features used in characterization of epileptic seizure dynamics can be found in recent studies $[27,30,42]$. In both seizure detection and prediction, the approaches are similar in terms of extracting features, but the goals are different. In seizure recognition/detection the goal is to detect the seizure onset as accurately as possible. Therefore, in seizure detection we try to differentiate between ictal and non-ictal time epochs, where ictal period (defined as an EEG event rather than a clinical event) can be identified by neurologists with some level of subjectivity based on visual analysis of EEG signals. On the other hand, seizure prediction aims to predict seizures taking into consideration very early signs of an upcoming seizure. We therefore focus on the differentiation of pre-ictal and inter-ictal periods. A pre-ictal period, however, has neither strict boundaries nor a definition and many studies in the literature have used a variety of features ([20] and references therein) to define a pre-ictal period. Changes in these features occur from several minutes up to several hours before a seizure onset $[5,13,19]$. Therefore, different prediction horizons are often used to asses the performance of proposed models. In addition to the evaluation of the seizure prediction system using different prediction horizons, a seizure prediction technique should also have a precise temporal resolution since the variation in the prediction horizon may simply make the proposed technique impractical as discussed in [33].

In the literature, studies often use either multiple features from a single channel or a single feature from multiple channels since data construction and data analysis techniques are restricted to two dimensions. For instance, in [30], seizure dynamics are analyzed solely on a specific recording, which represents the characteristics of a seizure well. Then the performance of various features from different domains on that particular signal is analyzed simultaneously. On the other hand, [27] analyzes multi-channel EEG data but assesses the performance of each feature one at a time. Furthermore, different studies extract different features and employ different algorithms to distinguish between seizure and non-seizure periods (e.g., [26] and references therein), which makes it difficult to compare the performance of features. An approach capable of simultaneously analyzing features would enable the performance comparison of the features on the same data using the same classifier. Simultaneous analysis of features is also important because it may consider linear or non-linear combinations of features. While a single feature may not be very effective in discriminating between epileptic periods, combinations of several features may well be [8].

We note that all techniques reviewed above focus on the spikes and sharp waves in each electrode via temporal, frequency, or wavelet domain analysis [3,11,31,41]. However, the underlying mechanism may also be captured via analyzing the signals from different electrodes jointly such as mul- tiway analysis techniques [1]. Multi-modality of the data enables one to include as many features as possible in the analysis, and combines the seizure recognition or prediction power of several features. Furthermore, since we can analyze multiple channels simultaneously, we do not have to make any prior assumption regarding the seizure localization and therefore select channels.

Multiway analysis techniques have been previously applied successfully in neuroscience. In [7], EEG data and data collected through experiments with different doses of a drug are arranged as a six-way array with modes: EEG, patients, doses, conditions, time, and channels. The analysis of the six-way dataset demonstrates that significant information is successfully extracted from a complex drug dataset by a multi-linear model rather than two-way models such as Principal Component Analysis (PCA) [14,15]. Multiway models have become more popular in neuroscience with the idea of decomposing EEG data into space-time-frequency components [25]. The three-way array constructed from multi-channel EEG data in [25] with modes time samples $\times$ frequency $\times$ channels is analyzed using a multi-linear component model called Parallel Factor Analysis model

(PARAFAC) [9]. Components extracted by PARAFAC are observed to demonstrate the temporal, spectral and spatial signatures of EEG. In another study [28], PARAFAC models with non-negativity constraints are used on Event-Related Potentials (ERP) to find the underlying structure of brain dynamics. In addition to the applications of multi-linear component models, multi-linear regression models have also been previously employed in neuroscience, e.g., in [24] for extracting the connection between EEG and functional Magnetic Resonance Imaging (fMRI) recordings .

In $[1,2]$, multi-channel EEG data is converted to a third order tensor $\underline{\mathbf{X}} \in \mathbb{R}^{I \times J \times K}$ by applying continuous wavelet transformation on the signal recorded at each channel. In this tensor, $x_{i j k}$ represents the square of the absolute value of wavelet coefficient at the $i$ th channel for $j$ th scale and the $k$ th time sample; in other words the power of the wavelet transformed data. Analysis of the tensor by PARAFAC [9] algorithm revealed the seizure localization.

Recently there has emerged a new direction in analyzing EEG recordings by building a synchronization graph that enables characterization of the pairwise correlations between electrodes using graph theoretical features over time [4,37]. In the spatio-temporal EEG graphs, nodes (vertices) represent the EEG channels and the edges (links) represent the level of neuronal synchronization between the different regions of the brain. This approach has been exploited in the analysis of various neuropsychiatric diseases including schizophrenia, autism, dementia, and epilepsy [37]. Within epilepsy research, evolution of certain graph features over time revealed better understanding of the interactions of the brain regions and the seizures. For instance, Schindler et al. analyzed the change in path lengths and clustering coefficients to highlight the evolution of seizures on epileptic patients [34], Kramer et al. considered the evolution of local graph features including betweenness centrality to explain the coupling of brain signals at seizure onset [17], and Douw et al. recently showed epilepsy in glioma patients was attributed to the theta band activity in the brain [6]. In [23], authors independently suggest a similar approach that combines tensor decompositions with graph theory. 
In this paper, we study how to build synchronization graphs in depth and present graph mining results to detect epileptic seizure both in temporal and spatial domains. The organization of the paper is as follows: in Section 2, we describe the epileptic EEG dataset, methodology to construct timeevolving EEG synchronization graphs, and the computation of global and local features on these graphs. In Section 3, we present results for seizure detection and seizure localization using the features described in Section 2. Finally, we provide an overview, discuss the results, and list extensions to this study, in Section 4.

\section{METHODOLOGY}

\subsection{Epileptic EEG Dataset}

Our dataset consists of scalp EEG recordings of 34 seizures from 15 patients. All the patients were evaluated with scalp video-EEG monitoring in the international 10-20 system (as described in [16]), magnetic resonance imaging (MRI), fMRI for language localization, and position emission tomography (PET). The data was collected in the Neurophysiology Laboratory of Yeditepe University Hospital in Istanbul, Turkey. All the patients had Hippocampal Sclerosis (HS) except one patient (IY) with Cortical Dysplasia (CD). After selective amygdalohippocampectomy, all the patients were seizure free. The patient information is provided in Table 1. For 10 patients, the seizure would onset from the left $(\mathrm{L})$, whereas for 5 patients the seizure would onset from the right (R).

Table 1: Patient Types. Almost all the patients exhibited hippocampal sclerosis (HS). There are two types of lateralizations in HS: left (L) and right (R). One patient (IY) exhibited cortical dysplasia (CD).

\begin{tabular}{ccc} 
Patient & Pathology & Lateralization \\
\hline \hline IY & CD & R \\
ZGU & HS & R \\
OB & HS & R \\
BMI & HS & R \\
FZE & HS & R \\
ADE & HS & L \\
GSE & HS & L \\
IP & HS & $\mathrm{L}$ \\
AIZ & HS & L \\
ATU & HS & L \\
MSO & HS & L \\
ABA & HS & L \\
DAK & HS & L \\
NT & HS & L \\
SUL & HS & L
\end{tabular}

One patient has one 30 minute recording, two patients have two 30 minute recordings, one patient has three 30 minute recordings, three patients have single 60 minute recordings, three patients have two 60 minute recordings, four patients have three 60 minute recordings, and one patient has five 60 minute recordings. The recordings include sufficient pre-ictal and post-ictal periods for the analysis. Two of the electrodes $\left(A_{1}\right.$ and $\left.A_{2}\right)$ were unused and $C_{z}$ electrode was used for referential montage that yielded 18-channel EEG recordings. Dr. Aykut-Bingöl's team diagnosed the initiation and the termination of each seizure and reported these periods as the ground truth for our analysis. An example of such a recording can be found in Fig. 2 in [36]. Seizures were 97 seconds long on average and their standard deviation was 121 seconds.

\subsection{Construction of EEG Synchronization Graphs}

Let $\mathbf{X}[i, m]$ denote the recorded EEG signal, where $i \in$ $\{1, \ldots, 18\}$ represents the index for the $i$ th electrode and $m \in\left[1, \ldots, f_{s} \times M\right]$ represents the time index, $f_{s}$ represents the sampling frequency, and $M$ is the duration of the recording in seconds. Sampling frequency, $f_{s}$, is either $200 \mathrm{~Hz}$ or $400 \mathrm{~Hz}$. We first decompose the signal in each electrode into the five traditional frequency bands $\delta(0.5-3.5 \mathrm{~Hz}), \theta(3.5-$ $7.5 \mathrm{~Hz}), \alpha(7.5-12.5 \mathrm{~Hz}), \beta(12.5-30 \mathrm{~Hz})$, and $\gamma(>30 \mathrm{~Hz})$ through appropriate digital band-pass filters $H_{f}(\omega)$, where $f$ represents the index for the frequency band. Next, we constructed epochs of equal lengths with $20 \%$ overlap between the preceding and following epochs. The number of epochs $N$ is equal to $1.25 M / L$, where $L$ is the duration of the epoch in the same time units.

Given the nature of these spatio-temporal recordings, we consider constructing time-evolving EEG Synchronization Graphs on the EEG datasets. A graph is constructed for each frequency band and epoch. The nodes represent the EEG electrodes and the edges represent a closeness relationship between the nodes in a given epoch. The main goal sought in the time-evolving EEG synchronization graphs is the ability to sense both the spatial and temporal changes in the network, yielding measures of change for the mining.

The selection of epoch length is significant in both the time-domain and frequency-domain analysis techniques.

While longer epochs provide better frequency resolution, far too lengthy epochs may not be stationary [40] and not allow rapid detections of changes in the network [21,22]. Therefore, shorter epoch lengths are generally preferred. However, extremely short epoch lengths may not allow the generation of meaningful graphs for mining. Therefore, a secondary goal of this research is to analyze and identify the effects of epoch lengths on the time-evolving EEG Synchronization Graphs. Note that if a wavelet-domain based measure is employed for the construction of the time-evolving graphs, these effects are reduced or eliminated. However, as the range of explored frequencies must be arbitrarily defined prior to the wavelet analysis, wavelet domain techniques do not allow the simultaneous exploration of the whole range of the EEG frequency spectrum.

For purposes of illustration, we construct simple timeevolving graphs on the EEG recording shown in Fig. 1. The graph edges are established based on the pair-wise relationships between the epoch data. Specifically, an edge between two distinct nodes $i$ and $k$, where $i, k \in\{1, \ldots, 18\}$, and $i \neq k$, is established if the pair-wise distance for the $n$th epoch, $d_{i, k}^{n}$, is less than the threshold $\tau$. A parametric search is performed to find optimal values for the threshold $\tau$. Further information about the search performed on various parameters to determine optimal values is provided in Section 3.

Several synchronization measures have been proposed as plausible options for $d_{i, k}^{n}$. We consider 3 different measures here, namely Correlation Coefficient [29], Phase Lag 


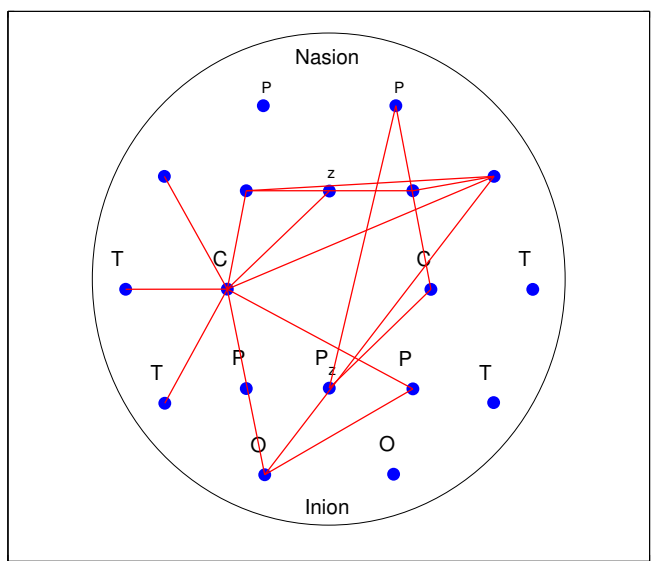

(a) Pre-ictal

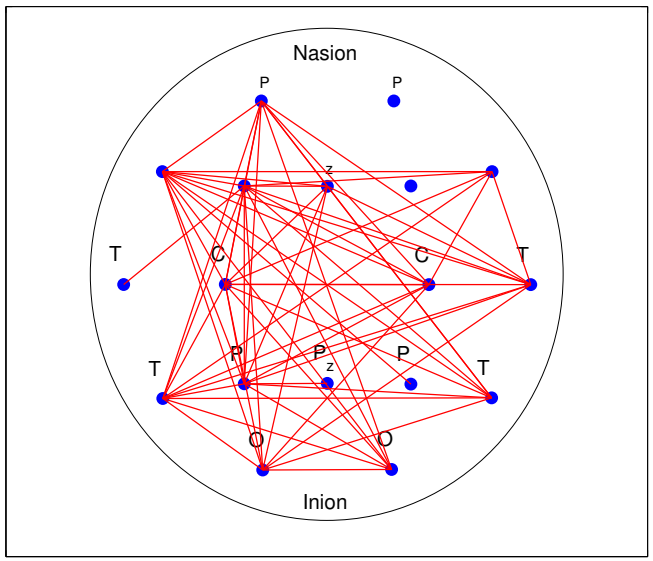

(b) Ictal

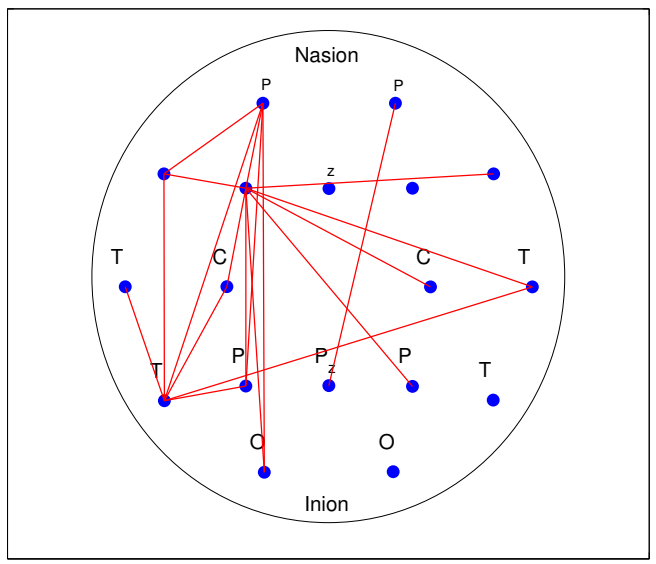

(c) Post-Ictal

Figure 1: Sample EEG Synchronization Graphs for pre-ictal, ictal, and post-ictal epochs. It is clearly seen that the ictal period has more coherence between different regions of the brain.

Index [38], and Phase Locking Value [18]. The three mea- sures are defined as follows:

$$
C C_{i, k}(n)=\frac{1}{L f_{s}} \frac{\left(\mathbf{x}_{i}^{n}-\overline{\mathbf{x}}_{i}^{n}\right)\left(\mathbf{x}_{k}^{n}-\overline{\mathbf{x}}_{k}^{n}\right)^{T}}{\sigma_{\mathbf{x}_{i}^{n}} \sigma_{\mathbf{x}_{k}^{n}}}
$$

$\overline{\mathbf{x}}_{i}^{n}$ and $\overline{\mathbf{x}}_{k}^{n}$ are the mean values of $\mathbf{x}_{i}^{n}$ and $\mathbf{x}_{k}^{n}$, respectively, $\sigma_{\mathbf{x}_{i}^{n}}$ is the standard deviation of $\mathbf{x}_{i}^{n}$,

$$
\begin{aligned}
P L V_{i, k}(n) & =\frac{1}{L f_{s}}\left|\sum_{m=1}^{L f_{s}} \exp \left(j\left(\phi_{i}^{n}(m)-\phi_{k}^{n}(m)\right)\right)\right| \\
P L I_{i, k}(n) & =\frac{1}{L f_{s}}\left|\sum_{m=1}^{L f_{s}} \operatorname{sgn}\left(\phi_{i}^{n}(m)-\phi_{k}^{n}(m)\right)\right|
\end{aligned}
$$

where $\phi_{i}^{n}=\arctan \left(\frac{\hat{\mathbf{x}}_{i}^{n}}{\mathbf{x}_{i}^{n}}\right)$ is the angle of the Hilbert transform $\hat{\mathbf{x}}_{i}^{n}$ of the signal $\mathbf{x}_{i}^{n}$. For correlation coefficient (Eq. 1), we define pair-wise distance as $d_{i, k}^{n}=1-C C_{i, k}(n)$. Note that smaller threshold values seek higher correlation between the electrodes, therefore yielding sparser graphs. Similarly, higher threshold values would establish an edge even if there is small correlation between the data, thereby yielding denser graphs.

\subsection{Feature Extraction from EEG Synchroniza- tion Graphs}

We extract 27 features from the EEG graph for each epoch. These features quantify the compactness, clusteredness, and uniformity of the graph. Compactness of a graph is measured by features such as average eccentricity, diameter, radius, and number of central points that are based on the path distances between the nodes within the graph. The clusteredness of a graph is measured by features such as average clustering coefficient and number of connected components that are based on how connected are the nodes and their neighbors with respect to each other. In addition, we also extract spectral graph features such as spectral radius and spectral gap using the eigenvalues of the adjacency, Laplacian, and normalized Laplacian matrices, that reveal additional clusteredness and compactness properties about the graphs. A complete list of the features used in this work and their definitions is listed in Table 2.

\subsection{Mining Global Graph Features for Tem- poral Clustering}

After computing the global graph features for each epoch, we build features matrices of dimensionality $N \times 27$, where $N$ is the number of epochs, for the sub-bands of the recording. In order to learn the structural differences within the temporal evolution of the epileptic seizures, we decided to separate the epochs into different clusters using unsupervised learning. We employed $k$-means clustering due to its successful record in bioinformatics applications [10]. $k$-means clustering is an iterative unsupervised learning method that partitions the samples into $k$ clusters based on their attributes and traits. The method first chooses $k$ random samples from the set and assigns them as the initial cluster centroids. Next, the remaining samples are assigned to the cluster whose centroid is the closest in terms of Euclidean distance. Finally, the cluster centroids are recomputed by averaging over all samples within the cluster and the process is repeated until the centroids do not change and withincluster residual sum of squares is minimized. Note that if significantly more recordings for each patient are available, 
Table 2: Description of EEG global graph features.

\begin{tabular}{|c|c|c|}
\hline Index & Feature Name & Description \\
\hline 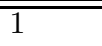 & Average Degree & Average number of edges per node \\
\hline 2 & Clustering Coefficient C & $\begin{array}{l}\text { Average of the ratio of the links a node's neighbors have in between } \\
\text { to the total number that can possibly exist }\end{array}$ \\
\hline 3 & Clustering Coefficient D & Same as feature 2 with node itself added to both numerator and denominator \\
\hline 4 & Average Eccentricity & $\begin{array}{l}\text { Average of node eccentricities, where the eccentricity of a node is the } \\
\text { maximum distance from it to any other node in the graph }\end{array}$ \\
\hline 5 & Diameter of graph & Maximum of node eccentricities \\
\hline 6 & Radius of graph & Minimum of node eccentricities \\
\hline 7 & Average Path Length & Average number of hops along the shortest paths for all possible pairs of nodes \\
\hline 8 & Giant Connected Component Ratio & $\begin{array}{l}\text { Ratio between the number of nodes in the largest connected component } \\
\text { in the graph and total the number of nodes }\end{array}$ \\
\hline 9 & Number of Connected Components & Number of clusters in the graph excluding the isolated nodes \\
\hline 10 & Average Connected Component Size & Average number of nodes per connected component \\
\hline 11 & $\%$ of Isolated Points & $\%$ of the isolated nodes in the graph, where an isolated node has a degree of 0 \\
\hline 12 & $\%$ of End Points & $\%$ of endpoints in the graph, where an endpoint has a degree of 1 \\
\hline 13 & $\%$ of Central Points & $\%$ of nodes within the graph whose eccentricity is equal to the graph radius \\
\hline 14 & Number of Edges & Number of edges between all nodes in the graph \\
\hline 15 & Spectral Radius & Largest eigenvalue of the adjacency matrix \\
\hline 16 & Adjacency Second Largest Eigenvalue & Second largest eigenvalue \\
\hline 17 & Adjacency Trace & Sum of the adjacency matrix eigenvalues \\
\hline 18 & Adjacency Energy & Sum of the square of adjacency matrix eigenvalues \\
\hline 19 & Spectral Gap & Difference between the magnitudes of the two largest eigenvalues \\
\hline 20 & Laplacian Trace & Sum of the Laplacian matrix eigenvalues \\
\hline 21 & Laplacian Energy & Sum of the square of Laplacian matrix eigenvalues \\
\hline 22 & Normalized Laplacian Number of 0's & Number of eigenvalues of the normalized Laplacian matrix that are 0 \\
\hline 23 & Normalized Laplacian Number of 1's & Number of eigenvalues of the normalized Laplacian matrix that are 1 \\
\hline 24 & Normalized Laplacian Number of 2's & Number of eigenvalues of the normalized Laplacian matrix that are 2 \\
\hline 25 & Normalized Laplacian Lower Slope & The sorted slopes of the line for the eigenvalues that are between 0 and 1 \\
\hline 26 & Normalized Laplacian Upper Slope & The sorted slope of the line for the eigenvalues that are between 1 and 2 \\
\hline 27 & Normalized Laplacian Trace & Sum of the normalized Laplacian matrix eigenvalues \\
\hline
\end{tabular}

supervised machine learning techniques will be more suitable to capture these differences. For the purpose of this study, we use $k$-means in an unsupervised setting, only using the ground truth labeling provided by the doctors to validate our clustering results.

\subsection{Spatial Analysis by Mining Local Graph Features}

The analysis of the global graph features indicated a critical change in their values during the transitionary period from pre-ictal to ictal, and from ictal to post-ictal. As a consequence of this outcome, we studied the change in the local graph features for individual nodes (electrodes) to determine whether we could localize the seizure to a particular part of the brain, or more specifically to a particular electrode. The intuition driving this hypothesis is that the electrode closest to the part of the brain in which the seizure onsets will record this change in brain activity before the other electrodes. The local graph features for the particular electrode will then indicate this sudden departure from regular behavior. We can verify our results based on the ground truth. To aid us in this objective, we utilize a Tucker3 tensor decomposition approach [39].

We construct the three-way array $\underline{\mathbf{X}} \in \mathbb{R}^{I \times J \times K}$ with modes: time samples, local graph features, and electrodes. We model $\underline{\mathbf{X}}$ using a $(P, Q, R)$-component Tucker3 model on a three-way array given by

$x_{i j k}=\sum_{p=1}^{P} \sum_{q=1}^{Q} \sum_{r=1}^{R} g_{p q r} a_{i p} b_{j q} c_{k r}+e_{i j k}$, where $\mathbf{A} \in \mathbb{R}^{I \times P}, \mathbf{B} \in \mathbb{R}^{J \times Q}$, and $\mathbf{C} \in \mathbb{R}^{K \times R}$ are the component matrices corresponding to the first, second, and third modes, respectively. $\underline{\mathbf{G}} \in \mathbb{R}^{P \times Q \times R}$ is the core array and $\underline{\mathbf{E}} \in \mathbb{R}^{I \times J \times K}$ contains the residuals. The illustration of a Tucker3 model on a three-way array is given in Fig. 2.

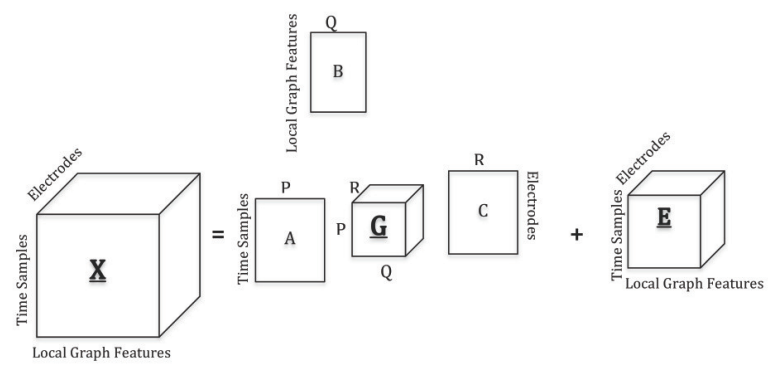

Figure 2: Tucker3 tensor decomposition. $(P, Q, R)$ component Tucker3 model, with a three-way array $\underline{\mathbf{X}} \in \mathbb{R}^{I \times J \times K}$ is modeled with component matrices $\overline{\mathbf{A}} \in \mathbb{R}^{I \times P}, \mathbf{B} \in \mathbb{R}^{J \times Q}$, and $\mathbf{C} \in \mathbb{R}^{K \times R}$ in the time samples (first), local graph features (second), and electrode (third) modes, respectively. $\underline{\mathbf{G}} \in \mathbb{R}^{P \times Q \times R}$ is the core array and $\underline{\mathbf{E}} \in \mathbb{R}^{I \times J \times K}$ contains the residuals. 
For each patient, we construct the three-way array $\underline{\mathbf{X}}$ with the modes: time samples within the seizure period, local graph features computed for each electrode, and the number of electrodes. We compute 8 local graph features listed in Table 3. We run the Tucker3 decomposition on this tensor.

We examine the plotting of the residual sum of squares vs. hoteling T-squared values [12] in the electrodes mode which show the outliers in the upper right corner (as shown in Fig. 3). These outliers are the electrodes that we consider as progenitors of the seizure, and use their location to determine the side of the brain where the seizure begins. The electrodes present towards the upper left or bottom left corners are not significant. In the figure, electrodes are named using the following nomenclature: " $[\mathrm{L}|\mathrm{C}| \mathrm{R}][1-8]$ identifier", where "L", "C", and "R" represent left, center, and right sides of the brain, respectively. The electrodes are numbered from 1-8 and each electrode is given a unique identifier.

\section{EXPERIMENTAL RESULTS}

We conducted a parametric search to determine the effect of parameters to capture the characteristics of the seizures. We tested four different epoch lengths that vary from a relatively short epoch length of 5 seconds to a longer duration of 12.5 seconds with 2.5 second increments. We tested five different threshold $(\tau)$ values for each synchronization cost function tested in our methodology. After obtaining the EEG graphs for each epoch in a particular frequency band in the recording, we extracted the graph features and constructed the data matrix to be clustered. In order to reduce the scale differences among the features, the data was normalized so that the features have zero mean and unit variance across the epochs. We tested four different $l$ values ranging between two and five considering pre-ictal, ictal, post-ictal, as well as the transitional periods between these stages. The result of $k$-means clustering is typically biased by the initial cluster centroids that are chosen randomly. In order to obtain unbiased results, we repeated the clustering 100 times and choose the clusters that were obtained most frequently as the final result. The resulting clusters are evaluated using F-measure and cluster entropies. F-measure is a function of precision $(P)$ and recall $(R)$ of a particular class type after retrieval and is given by $F=2 \frac{P R}{P+R}$. The $e n-$ tropy of a cluster is given by $E=-\sum_{k=1}^{K} \operatorname{Pr}\left(c_{k}\right) \log _{2} \operatorname{Pr}\left(c_{k}\right)$, where $\operatorname{Pr}\left(c_{k}\right)$ is the probability of a time epoch belonging to cluster $c_{k}$. For an ideal clustering, we want F-measure to be close to 1 and entropy to be close to 0 . Therefore, we combine these two metrics in a single one as $F / E$ where uniform clusters yield higher values. A total of 75 parameters are tested for each recording and we choose the clusters that yield the highest value as the representative result for that recording. Figure 4 displays clusters for a particular EEG recording. As can be noticed from the figure, the seizure time points are present within a single cluster, namely cluster 5 (marked in red), whereas the pre-ictal and post-ictal time points belong to various clusters, namely clusters $1-4$.

\subsection{Results and Interpretations}

In the following 2 sections, we present results for the seizure detection and seizure localization algorithms. Please note that in two patients with identifiers ATU_1 and ATU_2, and ABA_1, we could not detect and localize the seizures well. In these patients, we reviewed the seizure samples

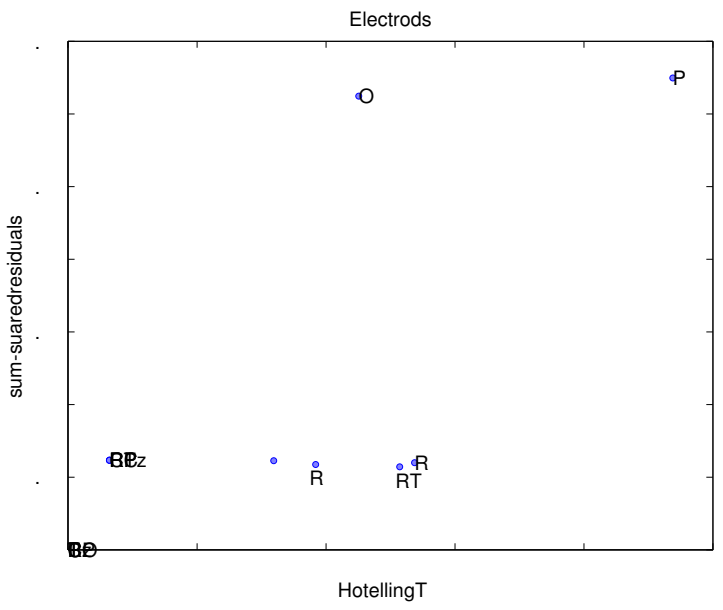

(a) NT_3

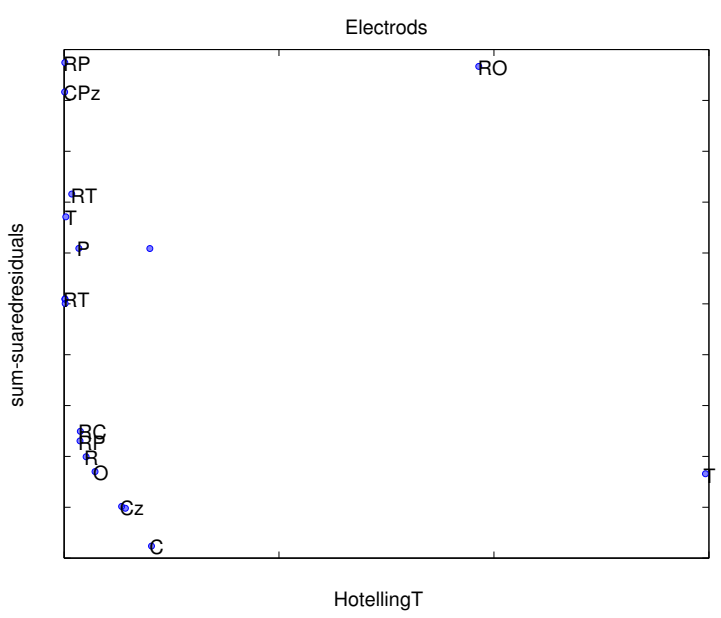

(b) FZE_4

Figure 3: Seizure localization. Figure shows two examples of seizure localization, for NT_3 the seizure onsets from the left, whereas for FZE_4 the seizure onsets from the right. In the case of NT_3, electrode P3 is chosen as the likely location for seizure onset this electrode is on the left side of the brain. In the case of FZE_4, electrode $\mathrm{O} 2$ is chosen as the likely location for seizure onset - this electrode is on the right side of the brain.

again and then we realized that we did not get the exact seizure periods. This result confirmed the veracity of our data analysis.

\subsubsection{Seizure Detection}

We found that our seizure detection algorithm was able to correctly detect the seizure in 30 of the 34 patient recordings for a success rate of $88.24 \%$. If we discount the recordings with identifiers ATU_1 and ATU_2, which had issues even with external video EEG information, our accuracy increases to $93.75 \%$.

We also present the seizure detection results based on 4 criteria: 
Table 3: Description of EEG local graph features

\begin{tabular}{ll} 
Index & Feature Name \\
\hline \hline 1 & Degree of node \\
2 & Clustering Coefficient C \\
3 & Clustering Coefficient D \\
4 & Clustering Coefficient E \\
5 & Eccentricity \\
6 & Eccentricity 90\% \\
7 & Closeness Centrality \\
8 & Betweenness Centrality
\end{tabular}

Description

Number of edges incident to the node

The ratio of the edges between the node's neighbors to the total number that can possibly exist

The ratio of the edges between the node's neighbors and the node itself to the total

number that can possibly exist

Same as clustering coefficient $\mathrm{C}$ but discounting the isolated nodes

Maximum shortest path length from the node to any other node in the graph

Maximum shortest path length from the node to $90 \%$ of the reachable nodes in the graph

Sum of the distances of the node from all other nodes in the graph determines its importance

Measures how many shortest paths between all pairs of nodes include the node

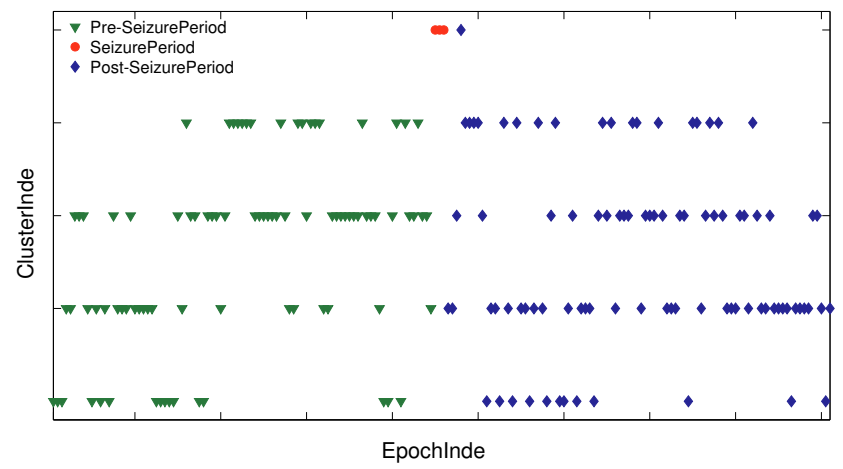

Figure 4: Clusters for an EEG recording. The seizure time points are all within one cluster (5) and are marked in red. The pre-ictal time points are marked in green, and the post-ictal time points are marked in blue.

1. Comparison of multiple frequency bands to a single frequency band consisting of the entire signal.

We found that considering the top $5 \mathrm{~F}$-score values for each patient recording, multiple frequency bands outperformed single frequency band in $72.35 \%$ of the patient recordings.

2. The frequency band with the most consistently high F-score values.

The $\theta$ frequency band had the best F-scores in $34.71 \%$ of the patient recordings.

3. The synchronization metric with the most consistently high F-score values.

The Phase Lag Index synchronization metric had the best $\mathrm{F}$-scores in $51.18 \%$ of the patient recordings.

4. The epoch length with the most consistently high Fscore values.

Epoch length of 12.5 seconds had the best F-scores in $45.29 \%$ of the patient recordings. As mentioned in Section 2.2, determining the optimal epoch length is crucial in constructing meaningful graphs on which mining techniques can be applied.

\subsubsection{Seizure Localization}

Table 4 presents the patient pathologies with the results of seizure localization. A "+" sign indicates correctly identified seizure, whereas a "-" sign indicates an incorrectly identified seizure. We were able to correctly localize the seizure in 26 of the 34 recordings leading to a $76.47 \%$ success rate. If we discount the recordings with identifiers ATU_1 and ATU_2, which had issues even with additional video EEG information, our accuracy increases to $81.25 \%$. We recognized that the clustering coefficient synchronization metric was responsible for almost all the incorrect localizations.

\section{DISCUSSION AND CONCLUSION}

Synchronization in the firing of neurons is one of the characteristics of epileptic seizure. In this work we modeled this phenomena by building synchronization graphs, as a method to quantify the correspondence among electrodes recording EEG signals. The electrodes form the nodes in the graph and an edge is inserted between these electrodes when their pair-wise synchronization is greater than a threshold. We extracted graph features computed over the entire graph (global features), and for individual nodes (local features), and used these features to analyze the data in the feature space to detect seizure localization in both spatial and temporal domains. We identified the free parameters in this model and ran an extensive parametric search to determine the best possible configurations. When evaluated against the ground truth provided by the medical team, our results were found to be encouraging, but have scope for further improvement. One possible improvement would be to extend the feature set to include more graph features and then run a feature selection algorithm.

As we mentioned in Section 2.1, patients considered in this study had undergone surgery thus allowing us to precisely determine the location of the seizure origin. In our future work we will expand our data to include patients without surgical treatment.

The time-evolving graphs can be enriched in two novel ways. First, given the time evolution of the graphs and uncertainty on the optimum epoch length, the epoch lengths can be chosen stochastically or adaptively to create different time scales. Given that the nodes are constant (i.e. electrodes or sensors remain the same), we can use a node as the common point of reference to integrate the data/knowledge at different scales. In other words we can use node/vertex based augmentation across different time scales. The information/data from different scales can also have different weights (importance). For example, if we are going to use 
Table 4: Seizure localization results. Table lists patient identifier, pathologies, and the result of the seizure localization. A + sign indicates a correctly localized seizure, whereas - sign indicates an incorrectly localized seizure.

Patients Pathology and Lateralization Seizure Localization

\begin{tabular}{|c|c|c|}
\hline IY & CD R & $\begin{array}{l}+ \\
-\end{array}$ \\
\hline $\mathrm{ZGU}$ & HS R & + \\
\hline $\mathrm{OB}$ & HS R & $\begin{array}{l}+ \\
+\end{array}$ \\
\hline BMI & HS R & $\begin{array}{l}+ \\
+ \\
+\end{array}$ \\
\hline FZE & HS R & $\begin{array}{l}+ \\
+ \\
+ \\
+ \\
-\end{array}$ \\
\hline ADE & HS L & + \\
\hline GSE & HS L & + \\
\hline IP & HS L & - \\
\hline AIZ & HS L & $\begin{array}{l}+ \\
- \\
\end{array}$ \\
\hline ATU & HS L & $\begin{array}{l}- \\
- \\
\end{array}$ \\
\hline MSO & HS L & $\begin{array}{l}+ \\
-\end{array}$ \\
\hline $\mathrm{ABA}$ & HS L & $\begin{array}{c}\text { none } \\
+ \\
-\end{array}$ \\
\hline DAK & HS L & $\begin{array}{l}+ \\
+ \\
+\end{array}$ \\
\hline $\mathrm{NT}$ & HS L & $\begin{array}{l}+ \\
+ \\
+ \\
\end{array}$ \\
\hline SUL & HS L & $\begin{array}{l}+ \\
+ \\
+\end{array}$ \\
\hline
\end{tabular}

global features to represent the data, weighted averages can be utilized. Assigning optimum weights to scales is another possible research problem that can be addressed as an extension to this work. Second, we can augment the edges with the "similarity vector" between a pair of nodes. This vector contains several "node" features extracted from the "signal" (not from the graphs) and each feature may have a different weight as well.

\section{Acknowledgments}

This work was supported in part by National Institutes of Health grants RO1 EB008016 and R01 DE019244. The authors would also like to acknowledge valuable inputs and discussions from Dr. Metin Erturkler and Selim Orhan.

\section{REFERENCES}

[1] E. Acar, C. Aykut-Bingol, H. Bingol, R. Bro, and B. Yener. Multiway analysis of epilepsy tensors. Bioinformatics, 23(13):i10-i18, 2007.
[2] E. Acar, C. A. Bingol, B. H., and Y. B. Computational analysis of epileptic focus localization. In Fourth IASTED Int. Conf. on Biomedical Engineering, pages 317-322, 2006.

[3] M. Akin. Comparison of wavelet transform and FFT methods in the analysis of EEG signals. Journal of Medical Systems, 26(3):241-247, 2002.

[4] E. Bullmore and O. Sporns. Complex brain networks: graph theoretical analysis of structural and functional systems. Nature Reviews Neuroscience, 10(3):186-198, 2009.

[5] M. D'Alessandro, G. Vachtsevanos, R. Esteller, J. Echauz, S. Cranstoun, G. Worrell, L. Parish, and L. B. A multi-feature and multi-channel univariate selection process for seizure prediction. Clinical Neurophysiology, 116:506-516, 2005.

[6] L. Douw, E. van Dellen, M. de Groot, J. J. Heimans, M. Klein, C. J. Stam, and J. C. Reijneveld. Epilepsy is related to theta band brain connectivity and network topology in brain tumor patients. BMC Neuroscience, 11(1):103, 2010.

[7] F. Estienne, N. Matthijs, D. L. Massart, P. Ricoux, and L. D. Multiway modeling of high-dimensionality electroencephalographic data. Chemometrics Intell. Lab. Systems, 58(1):59-72, 2001.

[8] I. Guyon and A. Elisseeff. An introduction to variable and feature selection. Journal of Machine Learning Research, 3(1157-1182), 2003.

[9] R. A. Harshman. Foundations of the PARAFAC procedure: Models and conditions for an explanatory multi-modal factor analysis. UCLA working papers in phonetics, 16:1-84, 1970.

[10] J. A. Hartigan. Clustering Algorithms. John Wiley and Sons, Inc., 99th edition, 1975.

[11] B. Hjorth. EEG analysis based on time domain properties. Electroencephalography and Clinical Neurophysiology, 29(3):306-310, 1970.

[12] H. Hotelling. The generalization of student's ratio. The Annals of Mathematical Statistics, 2(3):360-378, 081931.

[13] L. D. Iasemidis, D. Shiau, P. Pardalos, W. Chaovalitwongse, K. Narayanan, A. Prasad, K. Tsakalis, P. Carney, and J. Sackellares. Long-term prospective on-line real-time seizure prediction. Clinical Neurophysiology, 116:532-544, 2005.

[14] J. E. Jackson. Principal components and factor analysis: Part I - principal components. J. Quality Technology, 12:201-213, 1980.

[15] J. E. Jackson. Principal components and factor analysis: Part II - additional topics related to principal components. J. Quality Technology, 13:46-58, 1981.

[16] H. H. Jasper. The ten-twenty electrode system of the international federation. Electroencephalogr Clin Neurophysiol Suppl., 10:371-375, 1958.

[17] M. A. Kramer, E. D. Kolaczyk, and H. E. Kirsch. Emergent network topology at seizure onset in humans. Epilepsy Research, 79(2):173-186, 2008.

[18] J. P. Lachaux, E. Rodriguez, J. Martinerie, and V. F. J. Measuring phase synchrony in brain signals. Human Brain Mapping, 8(4):194-208, 1999. 
[19] Q. M. Le Van, J. Soss, V. Navarro, R. Robertson, M. Chavez, M. Baulac, and J. Martinerie. Preictal state identification by synchronization changes in long-term intracranial EEG recordings. Clinical Neurophysiology, 116:559-568, 2005.

[20] K. Lehnertz and B. Litt. The first international collaborative workshop on seizure prediction: summary and data description. Clinical Neurophysiology, 116:493-505, 2005.

[21] W. J. Levy. The effect of epoch length on the identification of changes in EEG power spectra. Anesthesiology, 65(3A):A539, 1986.

[22] W. J. Levy. The effect of epoch length on the identification of changes in EEG power spectra. Anesthesiology, 66(4):489-495, 1987.

[23] A. Mahyari and S. Aviyente. Identification of dynamic functional brain network states through tensor decomposition. In 39th IEEE International Conference on Acoustics, Speech, and Signal Processing (ICASSP 2014), 2014.

[24] E. Martinez-Montes, P. A. Valdes-Sosa, F. Miwakeichi, R. I. Goldman, and M. S. Cohen. Concurrent EEG/FMRI analysis by multiway partial least squares. NeuroImage, 22(3):1023-1034, 2004.

[25] F. Miwakeichi, E. Martnez-Montes, P. Valds-Sosa, N. Nishiyama, H. Mizuhara, and Y. Y. Decomposing EEG data into space-time-frequency components using parallel factor analysis. NeuroImage, 22(3):1035-1045, 2004.

[26] H. R. Mohseni, A. Maghsoudi, and M. B. Shamsollahi. Seizure detection in EEG signals: A comparison of different approaches. In 28th Annual Int. Conf. of the IEEE Engineering in Medicine and Biology Society, volume Supplement, pages 6724-6727, 2006.

[27] F. Mormann, T. Kreuz, C. Rieke, R. Andrzejak, A. Kraskov, P. David, C. Elger, and K. Lehnertz. On the predictability of epileptic seizures. Clinical neurophysiology, 116(3):569-587, 2005.

[28] M. Mørup, L. K. Hansen, C. S. Herrmann, J. Parnas, and S. M. Arnfred. Parallel factor analysis as an exploratory tool for wavelet transformed event-related EEG. NeuroImage, 29(3):938-947, 2006.

[29] P. L. Nunez, R. Srinivasan, A. F. Westdorp, R. S. Wijesinghe, D. M. Tucker, R. B. Silbersteine, and P. J. Cadusch. EEG coherency: I: statistics, reference electrode, volume conduction, laplacians, cortical imaging, and interpretation at multiple scales. Electroencephalography and Clinical Neurophysiology, 103(5):499-515, 1997.

[30] N. Päivinen, S. Lammi, A. Pitkänen, J. Nissinen, M. Penttonen, and T. Grönfors. Epileptic seizure detection: A nonlinear viewpoint. Computer methods and programs in biomedicine, 79(2):151-159, 2005.

[31] O. A. Rosso, M. T. Martin, and A. Plastino. Brain electrical activity analysis using wavelet-based informational tools. Physica A: Statistical Mechanics and its Applications, 313(3-4):587-608, 2002.

[32] M. E. Saab and J. Gotman. A system to detect the onset of epileptic seizures in scalp EEG. Clinical Neurophysiology, 116:427-442, 2005.

[33] B. Schelter, M. Winterhalder, H. Drentrup, J. Wohlmuth, J. Nawrath, A. Brandt, S. B. A., and
J. Timmer. Seizure prediction: The impact of long prediction horizons. Epilepsy Research, 73:213-217, 2007.

[34] K. A. Schindler, S. Bialonski, M.-T. Horstmann, C. E. Elger, and K. Lehnertz. Evolving functional network properties and synchronizability during human epileptic seizures. CHAOS: An Interdisciplinary Journal of Nonlinear Science, 18(3):033119, 2008.

[35] A. Shoeb, H. Edwards, J. Connolly, B. Bourgeois, S. T. Treves, and G. J. Patient-specific seizure onset detection. In 26th Int. Conf. of the IEEE Engineering in Medicine and Biology Society, volume 1, pages 419-422, 2004.

[36] S. J. M. Smith. EEG in the diagnosis, classification, and management of patients with epilepsy. $J$ Neurol Neurosurg Psychiatry, 76:ii2-i-i7, 2005.

[37] C. Stam and E. van Straaten. The organization of physiological brain networks. Clinical Neurophysiology, 2012.

[38] C. J. Stam, G. Nolte, and A. Daffertshofer. Phase lag index: Assessment of functional connectivity from multi channel EEG and MEG with diminished bias from common sources. Human Brain Mapping, 28:1178-1193, 2007.

[39] L. R. Tucker. The extension of factor analysis to three-dimensional matrices. In H. Gulliksen and N. Frederiksen, editors, Contributions to Mathematical Psychology., pages 110-127. Holt, Rinehart and Winston, New York, 1964.

[40] M. van de Velde, I. R. Ghosh, and P. J. M. Cluitmans. Context related artefact detection in prolonged EEG recordings. Computer Methods and Programs in Biomedicine, 60(3):183-196, 1999.

[41] M. Van Gils, A. Rosenfalck, S. White, P. Prior, J. Gade, L. Senhadji, C. Thomsen, I. R. Ghosh, R. M. Longford, and K. Jensen. Signal processing in prolonged EEG recordings during intensive care. IEEE Engineering in Medicine and Biology Magazine, 16(6):56-63, 1997.

[42] M. J. A. M. van Putten, T. Kind, F. Visser, and V. Lagerburg. Detecting temporal lobe seizures from scalp EEG recordings: A comparison of various features. Clinical neurophysiology, 116(10):2480-2489, 2005. 